\title{
A non-dominated sorting Differential Search Algorithm Flux Balance Analysis (ndsDSAFBA) for in silico multiobjective optimization in identifying reactions knockout
}

\begin{abstract}
Metabolic engineering is defined as improving the cellular activities of an organism by manipulating the metabolic, signal or regulatory network. In silico reaction knockout simulation is one of the techniques applied to analyse the effects of genetic perturbations on metabolite production. Many methods consider growth coupling as the objective function, whereby it searches for mutants that maximise the growth and production rate. However, the final goal is to increase the production rate. Furthermore, they produce one single solution, though in reality, cells do not focus on one objective and they need to consider various different competing objectives. In this work, a method, termed ndsDSAFBA (non-dominated sorting Differential Search Algorithm and Flux Balance Analysis), has been developed to find the reaction knockouts involved in maximising the production rate and growth rate of the mutant, by incorporating Pareto dominance concepts. The proposed ndsDSAFBA method was validated using three genome-scale metabolic models. We obtained a set of nondominated solutions, with each solution representing a different mutant strain. The results obtained were compared with the single objective optimisation (SOO) and multi-objective optimisation (MOO) methods. The results demonstrate that ndsDSAFBA is better than the other methods in terms of production rate and growth rate.
\end{abstract}

Keyword: Multi-objective evolutionary algorithms; Metabolic engineering; Flux balance analysis; Reaction knockout; Pareto dominance; Artificial intelligence; Bioinformatics 